\title{
QUOTIENT CLOSED METANILPOTENT FITTING CLASSES
}

\author{
T. R. BERGER, R. A. BRYCE and JOHN COSSEY
}

(Received 3 February 1984)

Communicated by $\mathbf{H}$. Lausch

\begin{abstract}
A Fitting class of finite soluble groups is one closed under the formation of normal subgroups and products of normal subgroups. It is shown that the Fitting classes of metanilpotent groups which are quotient group closed as well are primitive saturated formuations.
\end{abstract}

1980 Mathematics subject classification (Amer. Math. Soc.): 20 F 16.

In Section 7 of [2] we proved that a Fitting class $\mathscr{F}$ of finite metanilpotent groups which is quotient group closed is a primitive saturated formation provided that is generated by supersoluble groups. We are now able to remove this qualification.

THEOREM. A Fitting class of metanilpotent groups which is quotient group closed is a primitive saturated formation.

All groups mentioned here will be finite and metanilpotent. We assume that the reader is familiar with the ideas and methods of [1], [2] and [3] most of which are by now standard. Accordingly we suppress arguments which are variations on those in the works cited.

Let $\mathscr{F}$ be a metanilpotent and Q-closed Fitting class. In order to prove the theorem it suffices to show that

(1) if $p, q$ are primes and if $\mathscr{F} \cap \mathscr{S}_{p} \mathscr{S}_{q}$ contains a non-nilpotent group then $\mathscr{S}_{p} \mathscr{S}_{q} \subseteq \mathscr{F}$.

() 1985 Australian Mathematical Society $0263-6115 / 85 \$ A 2.00+0.00$ 
For, let $\mathscr{F}_{p}$ be the Fitting formation generated by the automizers of all $p$-chief factors of all groups in $\mathscr{F}$. Note that $\mathscr{F}_{p}$ contains a non-trivial $q$-group for some prime $q$ if and only if some group in $\mathscr{F}$ has a $p$-chief factor whose automizer has order divisible by $q$. Let $\mathscr{F}_{0}$ denote the local formation defined by the $\mathscr{F}_{p} . \mathscr{F}_{0}$ is a primitive saturated formation [4], and $\mathscr{F} \subseteq \mathscr{F}_{0}$. We show that $\mathscr{F}=\mathscr{F}_{0}$. If we suppose, to the contrary, that $\mathscr{F} \neq \mathscr{F}_{0}$ then we may select a group $G$ in $\mathscr{F}_{0} \backslash \mathscr{F}$ of least order. Since $\mathscr{F}_{0}$ is normal subgroup and normal product closed $G / F(G)$ is a $q$-group for some prime $q$; and $F(G)$ is a $p$-group for some prime $p$ because if

$$
F(G)=S_{1} \times S_{2} \times \cdots \times S_{r}
$$

is the Sylow decomposition of $F(G)$ then, $\mathscr{F}_{0}$ being Q-closed and $G$ mininal, $G / S_{i} \in \mathscr{F}(1 \leqslant i \leqslant r)$ and $G \in \mathrm{s}_{n}\left\{G / S_{1} \times G / S_{2} \times \cdots \times G / S_{r}\right\} \subseteq \mathscr{F}$, a contradiction. Moreover $G$ is not nilpotent since $\mathscr{F}$ contains all $p$-groups for primes $p$ dividing the order of groups in $\mathscr{F}$. Hence $\mathscr{F}_{p}$ contains a non-trivial $q$-group and so there is a group $L$ in $\mathscr{F}$ with a $p$-chief factor whose automizer has order divisible by $q$. If $L_{0} / F(L)$ is the Sylow $q$-subgroup of $L / F(L)$ then $L_{0} \in \mathscr{F}$ and, if $T$ is the Hall $p^{\prime}$-subgroup of $F(L)$, then $L_{0} / T \in \mathscr{F}$. But $L_{0} / T$ is not nilpotent and belongs to $\mathscr{F} \cap \mathscr{S}_{p} \mathscr{S}_{q}$. By (1), therefore, $G \in \mathscr{S}_{p} \mathscr{S}_{q} \subseteq \mathscr{F}$, a contradiction to $\mathscr{F} \neq \mathscr{F}_{0}$. This completes the reduction of the Theorem to (1).

We can further reduce the class of groups we need to consider. For different primes $p, q$ let $\mathscr{U}(p, q)$ denote the class of groups of the form $P C$ where $P$ is a normal $p$-subgroup and $C$ a subgroup of order $q$.

(2) If $\mathscr{U}(p, q) \subseteq \mathscr{F}$ then $\mathscr{S}_{p} \mathscr{S}_{q} \subseteq \mathscr{F}$.

To prove (2) suppose that $G=A B$ with $A \unlhd G, A \in \mathscr{S}_{p}, B \in \mathscr{S}_{q}$. If $B$ is not cyclic then it may be written as a product of normal subgroups $B_{1}, B_{2}$. By induction on $|B|$ we conclude that

$$
A B \in \mathrm{N}_{0}\left\{A B_{1}, A B_{2}\right\} \subseteq \mathscr{F} .
$$

If $B$ is cyclic and $|B|=q$ then $G \in \mathscr{F}$ by hypothesis, so it remains to consider the case when $B$ is cyclic of order $q^{\alpha}$ with $\alpha>1$. The group $W=C_{q}$ wr $C_{q^{\alpha-1}}$ has a subgroup isomorphic to $B$, and we identify them. Let

$$
T=A \operatorname{twr}_{B} W
$$

the reader is referred to page 227 of [3] for an account of the twisted wreath product, and for the notation used there. Since $W$ is a product of subnormal (cyclic) subgroups of order less than $q^{\alpha}$ we have, by induction, that $T \in \mathscr{F}$. It follows that

$$
\left(\left.A\right|^{W}\right) B \in \mathscr{F}
$$

By Lemma 2.3 of [3]

$$
\left.\left.A\right|^{W}\right|_{B} \cong A \times K
$$


where $K$ admits $B$. Consequently

$$
A B \cong\left(\left.A\right|^{W}\right) B / K \in \mathrm{Q} \mathscr{F}=\mathscr{F} .
$$

This completes the induction and the proof of (2).

The proof of (1) is further reduced by another proposition: here $D_{p}^{q}$ is the unique (up to isomorphism) group which has a unique minimal normal subgroup, a $p$-group, with a complement of order $q$.

(3) If $D_{p}^{q} \in \mathscr{F}$ then $\mathscr{U}(p, q) \subseteq \mathscr{F}$.

The proof of this result uses the following invariant $\gamma(H)$ associated with a group $H$. Let $\mathfrak{A}_{p}$ denote the class of elementary abelian $p$-groups. Denote by $\mathfrak{A}_{p}(H)$ the smallest normal subgroup of $H$ whose factor group is an elementary abelian $p$-group, and by $\left(\mathfrak{A}_{p}\right)^{\delta}(H)$ its $\delta$ th iterate:

$$
\left(\mathfrak{A}_{p}\right)^{0}(H)=H \quad \text { and } \quad\left(\mathfrak{A}_{p}\right)^{\delta}(H)=\mathfrak{A}_{p}\left(\left(\mathfrak{A}_{p}\right)^{\delta-1}(H)\right), \quad \delta \geqslant 1 .
$$

Let $\delta$ be the greatest non-negative integer for which

$$
\left(\mathfrak{A}_{p}\right)^{\delta}\left(o_{p}(H)\right) \neq 1,
$$

and let $\varepsilon$ be the least positive integer for which

$$
\left[\left(\mathfrak{A}_{p}\right)^{\delta}\left(O_{p}(H)\right), \varepsilon O_{p}(H)\right]=1 .
$$

(As usual if $X, Y$ are subgroups of a group, $[X, Y]$ is the subgroup generated by all commutators $[x, y], x \in X, y \in Y ;[X, 1 Y]=[X, Y]$ and $[X, \varepsilon Y]=$ [[ $X,(\varepsilon-1) Y], Y]$ for $\varepsilon>1$.)

The invariant we use is the ordered pair

$$
\gamma(H)=(\delta, \varepsilon) .
$$

If we assume, contrary to the assertion (3), that $\mathscr{U}(p, q) \nsubseteq \mathscr{F}$, then there exists a group $G \in \mathscr{U}(p, q) \backslash \mathscr{F}$ for which $\gamma(G)$ is minimal in the lexicographical ordering of pairs. Note first that $\gamma(G) \geqslant(1,1)$. For, $\gamma(G)<(1,1)$ means that $P=O_{p}(G)$ is elementary abelian whence, if $G=P C$ with $|C|=q, P=P_{1} \times P_{2}$ $\times \cdots \times P_{s}$ where each $P_{i}$ is minimal normal in $G$; and then $P_{i} C \cong D_{p}^{q}$ or $P_{i} C \cong C_{p} \times C_{q}$. Since $D_{p}^{q}$ and $C_{p} \times C_{q}$ both belongs to $\mathscr{F}$ under the hypothesis of (3) we conclude that

$$
G \in \mathrm{S}_{n} \mathrm{~N}_{0}\left\{D_{p}^{q}, C_{p} \times C_{q}\right\} \subseteq \mathscr{F}
$$

Hence $\gamma(G) \geqslant(1,1)$.

The chain of lemmas we need to establish (3) begins now, and the notation of the last paragraph is maintained: $G$ is a minimal counterexample to the truth of (3), $O_{P}(G)=P$ and $G=P C$ where $|C|=q$. In what follows contructions involving several isomorphic copies of given groups abound. The following useful 
convention therefore will be employed, usually without further comment: isomorphic copies of a group $H$ will be denoted by subscripts, thus $H_{1}, H_{2}$ for example with $h_{1} \mapsto h, h_{2} \mapsto h$ being isomorphisms.

(4) If $K=A H$ where $A \unlhd K, A \in \mathfrak{A}_{p}$ and $H \in \mathscr{F}$, then $K \in \mathscr{F}$.

For, a semi-direct product $X=A_{1} H_{1}$ may be formed using the action of $H_{1}$ on $A_{1}$ inherited from that of $H$ on $A$ :

$$
a_{1}^{h_{1}}=\left(a^{h}\right)_{1} .
$$

By Lemma 5.1 of [2], $X \in \mathscr{F}$. However the mapping $X \rightarrow K$ defined by $h_{1} a_{1} \rightarrow h a$ is an onto homomorphism, so $K \in \mathscr{F}$ also.

(5) Suppose that $H \in \mathscr{F} \cap \mathscr{U}(p, q)$, that $A$ is a p-group on which $H$ acts as a group of operators, and that $\gamma(A)<\gamma(G)$. Then the semidirect product $A H \in \mathscr{F}$.

Let $H=Q D$ where $Q=O_{p}(H)$ and $|D|=q$. The proof of (5) is by induction on the smallest positive integer $r$ for which

$$
[A, r Q]=1 \text {. }
$$

If $r=1$ then $A H \in \mathrm{s}_{n}\{H \times A D\} \subseteq \mathscr{F}$, since $H \in \mathscr{F}$ by hypothesis and $A D \in \mathscr{F}$ by the minimality of $\gamma(G)$. So suppose $r>1$.

Write $\mathfrak{B}$ for the variety generated by the group $A Q$, and let $F$ be the $\mathfrak{B}$-product of $A_{1}$ and $Q_{1}$ :

$$
F=A_{1}{ }^{*} Q_{1} .
$$

(See Hanna Neumann [5], page 35 for a definition of verbal product.) There is a homomorphism $\alpha$ from $F$ onto $A Q$ whose restrictions to $A_{1}$ and $Q_{1}$ are, respectively, $a_{1} \mapsto a, x_{1} \mapsto x$. We define an action of $C_{1} \times C_{1}$ on $F$ as follows:

$$
a_{1}^{\left(c_{1}, c_{1}^{\prime}\right)}=\left(a^{c}\right)_{1}, \quad x_{1}^{\left(c_{1}, c_{1}^{\prime}\right)}=\left(x^{c^{\prime}}\right)_{1}, \quad a \in A, x \in Q .
$$

If $\bar{C}=\left\{\left(c_{1}, c_{1}\right): c \in C\right\}$ then $\alpha$ extends to a homomorphism $\bar{\alpha}: F \vec{C} \rightarrow A H$ via

$$
\left(c_{1}, c_{1}\right) \bar{\alpha}=c .
$$

Put $L=\operatorname{ker} \alpha(=\operatorname{ker} \bar{\alpha})$ and observe that $L \leqslant\left[A_{1}, Q_{1}\right]$.

Since $[A, r Q]=1$ we have $\left[A_{1}, r Q_{1}\right] \leqslant L$; and if $\gamma(A)=\left(\delta^{\prime}, \varepsilon^{\prime}\right)$ then $\left[\left(\mathfrak{A}_{p}\right)^{\delta^{\prime}}\left(A_{1}\left[A_{1}, Q_{1}\right]\right), \varepsilon^{\prime} A_{1}\right] \leqslant L$. Hence if $N$ is the normal closure in $F$ of $\left[A_{1}, r Q_{1}\right]$ and $\left[\left(\mathfrak{A}_{p}\right)^{\delta^{\prime}}\left(A_{1}\left[A_{1}, Q_{1}\right]\right), \varepsilon^{\prime} A_{1}\right]$ then $N \leqslant L$; also $N$ admits $C_{1} \times C_{1}$.

In what follows the convenient notation $X \in \mathscr{F}(\bmod Y)$ means that $Y$ is a normal subgroup of $X$ and $X / Y \in \mathscr{F}$. We have

$$
\begin{array}{ll}
{\left[A_{1}, Q_{1}\right] Q_{1}\left(1 \times C_{1}\right) \in \mathscr{F}(\bmod N),} & \text { by induction on } r ; \\
{\left[A_{1}, Q_{1}\right] A_{1}\left(C_{1} \times 1\right) \in \mathscr{F}(\bmod N),} & \text { by the minimality of } \gamma(G) ;
\end{array}
$$

whence

$$
\begin{aligned}
& F\left(1 \times C_{1}\right) \in \mathrm{N}_{0}\left\{F,\left[A_{1}, Q_{1}\right] Q_{1}\left(1 \times C_{1}\right)\right\} \subseteq \mathscr{F}(\bmod N), \\
& F\left(C_{1} \times 1\right) \in \mathrm{N}_{0}\left\{F,\left[A_{1}, Q_{1}\right] A_{1}\left(C_{1} \times 1\right)\right\} \subseteq \mathscr{F}(\bmod N) ;
\end{aligned}
$$


and consequently $(F / N)\left(C_{1} \times C_{1}\right) \in \mathrm{N}_{0} \mathscr{F}=\mathscr{F}$ so that $(F / N) \bar{C} \in \mathrm{s}_{n} \mathscr{F}=\mathscr{F}$. Finally $A H \in \mathrm{Q} \mathscr{F}-\mathscr{F}$, completing the induction and the proof of (5).

(6) Suppose that $K \in \mathscr{U}(p, q)$ has a subgroup $C$ of order $q$ and p-subgroups $Q, R$ normalized by $C$ and that

(i) $Q C, R C \in \mathscr{F}$,

(ii) $\gamma([Q, R])<\gamma(G)$, and

(iii) $Q$ and $R$ together generate $O_{p}(K)$.

Then $K \in \mathscr{F}$.

This result is a corollary of (5) and its proof is a re-play of that of (5). Consider a verbal product $X=Q_{1}{ }_{\mathfrak{B}} R_{1}$, where $\mathfrak{B}=\operatorname{var} O_{p}(K)$, and the natural homomorphism $\alpha: X \rightarrow O_{p}(K)$ given by $x_{1} \mapsto x, y_{1} \mapsto y(x \in Q, y \in R)$. Let $C_{1} \times C_{1}$ act on $X$ by

$$
x_{1}^{\left(c_{1}, c_{1}^{\prime}\right)}=\left(x^{c}\right)_{1}, \quad y_{1}^{\left(c_{1}, c_{1}^{\prime}\right)}=\left(y^{c^{\prime}}\right)_{1} .
$$

Again if $\bar{C}=\left\{\left(c_{1}, c_{1}\right): c \in C\right\}$ then $\alpha$ extends to a homomorphism $\bar{\alpha}: X \bar{C} \rightarrow K$ via

$$
\left(c_{1}, c_{1}\right) \bar{\alpha}=c .
$$

Let $\gamma([Q, R])=\left(\delta^{\prime}, \varepsilon^{\prime}\right)$. Then

$$
\left[\left(\mathfrak{A}_{p}\right)^{\delta^{\prime}}\left(\left[Q_{1}, R_{1}\right]\right), \varepsilon^{\prime}\left[Q_{1}, R_{1}\right]\right] \leqslant \operatorname{ker} \bar{\alpha}
$$

and so $N$, the normal closure in $X$ of $\left[\left(\mathfrak{A}_{p}\right)^{\delta^{\prime}}\left(\left[Q_{1}, R_{1}\right]\right), \varepsilon^{\prime}\left[Q_{1}, R_{1}\right]\right]$ is also contained in ker $\bar{\alpha}$; and it admits $C_{1} \times C_{1}$. Now

$$
\left[Q_{1}, R_{1}\right] Q_{1}\left(C_{1} \times 1\right) \in \mathscr{F}(\bmod N), \quad\left[Q_{1}, R_{1}\right] R_{1}\left(1 \times C_{1}\right) \in \mathscr{F}(\bmod N)
$$

by (5); then

$$
X\left(C_{1} \times 1\right) \in \mathrm{N}_{0}\left\{X,\left[Q_{1}, R_{1}\right] Q_{1}\left(C_{1} \times 1\right)\right\} \subseteq \mathscr{F}(\bmod N)
$$

and

$$
X\left(1 \times C_{1}\right) \in \mathrm{N}_{0}\left\{X,\left[Q_{1}, R_{1}\right] R_{1}\left(1 \times C_{1}\right)\right\} \subseteq \mathscr{F}(\bmod N)
$$

so

$$
X \bar{C} \in \mathrm{s}_{n} \mathrm{~N}_{0}\left\{X\left(C_{1} \times 1\right), X\left(1 \times C_{1}\right)\right\} \subseteq \mathscr{F}(\bmod N) .
$$

Finally $K \in Q^{\prime}\{X \bar{C} / N\} \subseteq \mathscr{F}$ as required.

(7) If $H$ is a group with an elementary abelian normal p-subgroup $T$ then there exists a group $W=W(H, T)$ with the following properties.

(i) $W$ has an elementary abelian normal p-subgroup $A$, which we call the base group of $W$, with a complement $\bar{H}$ in $W$ isomorphic to $H / T$.

(ii) As $Z_{p} \bar{H}$-module $A$ is injective.

(iii) There is an embedding $\eta: H \rightarrow W$ for which $W=A(H \eta)$ and $A \cap H \eta=T \eta$.

There are many ways of constructing such a group $W$. For example if $W=T$ wr $H / T$ then the Krasner-Kaloujnine embedding [5, page 46] of $H$ into $W$ 
has the desired properties, and $A$, the base group of $W$ is a direct sum of copies of the regular module for $\bar{H}=H / T$, therefore certainly injective. We will suppose whenever convenient that $H$ and $H \eta$ are identified.

(8) Let $T=\left(\mathfrak{A}_{p}\right)^{\delta}(P)$. Then $W(G, T) \in \mathscr{F}$ and $\left(\mathfrak{A}_{p}\right)^{\delta+1}(W(G, T))=1$.

For, $\bar{G} \cong G / T \in \mathscr{F}$ by the minimality of $\gamma(G)$ and then $W(G, T) \in \mathscr{F}$ by Lemma 5.1 of [2]. Also $\left(\mathfrak{A}_{p}\right)^{\delta}(W(G, T)) \in \mathfrak{A}_{p}$ so the other assertion is immediate.

Proof of (3). Write

$$
V= \begin{cases}T, & \text { if } \varepsilon=1, \\ {[T,(\varepsilon-1) P],} & \text { if } \varepsilon>1,\end{cases}
$$

and define $Y=W(W(G, T), V)$. (Here we are using the convenience of having $G \leqslant W(G, T)$.) Let $A$ be the base group of $Y$. By Sylow's Theorem we may suppose that $C \leqslant \bar{G}$, replacing $\bar{G}$ by one of its conjugates if necessary.

Now $\mathrm{C}_{Y}(V) \geqslant O_{p}(Y)$ and therefore, as $Z_{p} \bar{W}$-module, $V$ has an automorphism induced by a non-trivial element $c$ of $C$. Since $A$ is injective as $Z_{p} \bar{W}$-module there is an automorphism of $A$ agreeing on $V$ with the action of $c$. It follows that there is an action of $C_{1}$ on $Y$, and therefore a semi-direct product $Z=Y C_{1}$, satisfying $\left[\bar{W}, C_{1}\right] \leqslant A$ and $v^{d_{1}}=v^{d}, d \in C$.

By (8), and Lemma 5.1 of [2], $Y \in \mathscr{F}$ and therefore $Z \in \mathscr{F}$.

In the direct product $Z \times G_{2}$ consider the elements $\sigma=\left(c, c_{2}\right), \sigma^{\prime}=\left(c_{1}, c_{1}\right)$, and the subgroups $\Delta=O_{p}(Z) P_{2}\left\langle\sigma, \sigma^{\prime}\right\rangle$, and $\Gamma=\left\{\left(v, v_{2}\right): v \in V\right\}$. Plainly $\Gamma \unlhd \Delta$.

We claim that $\Delta / \Gamma \in \mathscr{F}$. To see this observe that

$$
\Delta \in \mathrm{N}_{0}\left\{O_{p}(Z) P_{2}\left\langle\sigma^{-1} \sigma^{\prime}\right\rangle, O_{p}(Z) P_{2}\langle\sigma\rangle\right\}
$$

so it suffices to show that $O_{p}(Z) P_{2}\left\langle\sigma^{-1} \sigma^{\prime}\right\rangle \in \mathscr{F}(\bmod \Gamma)$ and $O_{p}(Z) P_{2}\langle\sigma\rangle \in \mathscr{F}$ $(\bmod \Gamma)$. The first of these is easy:

$$
\begin{aligned}
O_{p}(Z) P_{2}\left\langle\sigma^{-1} \sigma^{\prime}\right\rangle & \in \mathrm{N}_{0}\left\{O_{p}(Z)\left\langle\sigma^{-1} \sigma^{\prime}\right\rangle, P_{2}\right\} \\
& \subseteq \mathrm{N}_{0} \mathrm{~s}_{n}\left\{Z, P_{2}\right\} \subseteq \mathscr{F},
\end{aligned}
$$

and this remains true modulo $\Gamma$. The second comes as follows. Let $\bar{P}=\left\{\left(x, x_{2}\right)\right.$ : $x \in P\}$. Since $\bar{P}$ admits $\sigma$ and since $\gamma(\bar{P} / \Gamma)<\gamma(G),(\bar{P} / \Gamma)\langle\sigma\rangle \in \mathscr{F}$. Also

$$
O_{p}(W(G, T))\langle\sigma\rangle \cong W(G, T) \in \mathscr{F}, \quad \text { by }(8) .
$$

Note too that

$$
\begin{aligned}
{\left[O_{p}(W(G, T)), \bar{P}\right] } & =\left[O_{p}(W(G, T)), P\right] \\
& \leqslant \mathfrak{A}_{p}\left(O_{p}(W(G, T))\right)
\end{aligned}
$$

and consequently by $(8)$ that $\gamma\left(\left[O_{p}(W(G, T)), \bar{P}\right]\right)<\gamma(G)$. It then follows from (6) that $\left(O_{p}(W(G, T)) P_{2} / \Gamma\right)\langle\sigma\rangle \in \mathscr{F}$. However

$$
O_{p}(Z) P_{2}\langle\sigma\rangle=A O_{p}(W(G, T)) P_{2}\langle\sigma\rangle
$$


which is in $\mathscr{F}(\bmod \Gamma)$ by (4). This completes the verification that $\Delta / \Gamma \in \mathscr{F}$.

We conclude, therefore, that

$$
\left(O_{p}(Z) P_{2} / \Gamma\right)\left\langle\sigma^{\prime}\right\rangle \in \mathrm{s}_{n} \mathscr{F}=\mathscr{F} .
$$

But this yields

$$
\left(A P_{2} / \Gamma\right)\left\langle\sigma^{\prime}\right\rangle \in \mathrm{s}_{n} \mathscr{F}=\mathscr{F} .
$$

By Maschke's Theorem $A \Gamma / \Gamma$ is completely reducible for the action of $\left\langle\sigma^{\prime}\right\rangle$; in particular $P_{2} \Gamma / \Gamma \cap A \Gamma / \Gamma$ is complemented in $A \Gamma / \Gamma$ by a normal subgroup of $\left(A P_{2} / \Gamma\right)\left\langle\sigma^{\prime}\right\rangle$. Hence

$$
G \cong P_{2}\left\langle\sigma^{\prime}\right\rangle \in \mathrm{Q}\left\{\left(A P_{2} / \Gamma\right)\left\langle\sigma^{\prime}\right\rangle\right\} \subseteq \mathscr{F} .
$$

This contradiction to the choice of $G$ concludes the proof of (3).

It remains to observe that if $\mathscr{F} \cap \mathscr{S}_{p} \mathscr{S}_{q}$ contains a non-nilpotent group then it contains $D_{p}^{q}$. For, if $H$ is a non-nilpotent group in $\mathscr{F} \cap \mathscr{S}_{p} \mathscr{S}_{q}$ of minimal order then $\Phi(H)=1$ so, in particular, $O_{p}(H)$ is elementary. Moreover if $D$ is a Sylow $q$-subgroup of $H$ then $D$ is cyclic and of order $q ; \mathrm{C}_{D}\left(O_{p}(H)\right)=1$; and $O_{p}(H)$ is irreducible for $D$. But then

$$
D_{p}^{q} \cong H \in \mathscr{F}
$$

as required.

\section{References}

[1] R. A. Bryce and John Cossey, 'Fitting formations of finite soluble groups', Math. Z. 127 (1972), 217-223.

[2] R. A. Bryce and John Cossey, 'Metanilpotent Fitting classes', J. Austral. Math. Soc. 12 (1974), 295-304.

[3] R. A. Bryce and John Cossey, 'Subgroup closed Fitting classes are formations, Math. Proc. Cambridge Philos. Soc. 91 (1982), 225-258.

[4] T. O. Hawkes, 'Skeletal classes of soluble groups', Arch. Math. 22 (1971), 577-589.

[5] Hanna Neuman, Varieties of groups (Ergebnisse der Mathematik und ihrer Grenzgebiete, Springer-Verlag, Berlin, Heidelberg, New York, 1967).

School of Mathematics

University of Minnesota

Minneapolis, Minnesota 55455

U.S.A.
Department of Mathematics Faculty of Science Australian National University

P.O. Box 4 Canberra, A.C.T. 2601 